\title{
Empirically Derived Dietary Patterns, Diet Quality Scores, and Markers of Inflammation and Endothelial Dysfunction
}

\author{
Linda M. Oude Griep • Huifen Wang • Queenie Chan
}

Published online: 19 April 2013

(C) Springer Science+Business Media New York 2013

\begin{abstract}
Atherosclerosis is one of the most important contributors to the global burden of cardiovascular diseases. With the recognition of atherosclerosis as an inflammatory disease, nutrition research interest has expanded toward the role of dietary patterns in the prevention of atherosclerosis primarily focused on associations with early inflammatory markers. This review summarizes the latest evidence from January 2010 until January 2013 of eight observational studies on the associations between empirically derived dietary patterns and diet quality scores with markers of inflammation and endothelial function. Overall, results of recently published cohort studies support those of previously published cross-sectional studies suggesting that consuming a healthy type of diet characteristically abundant in fruits and vegetables is associated with lower concentrations of Creactive protein and other inflammatory markers. Unfavorable associations were found between eating a western dietary pattern high in meat and inflammatory markers. Different statistical approaches of deriving dietary patterns were applied in these studies and most of them lacked in reporting types and absolute intakes of foods and/or food groups. Future prospective cohort studies are needed to evaluate long-term associations between dietary patterns and changes in inflammatory markers by comparing various
\end{abstract}

\footnotetext{
L. M. Oude Griep · Q. Chan

Department of Epidemiology and Biostatistics,

School of Public Health, St Mary's Campus,

Imperial College London, London, UK

H. Wang

Jean Mayer USDA Human Nutrition Research Center on Aging,

Tufts University, Boston, MA, USA

L. M. Oude Griep $(\bowtie)$

Department of Epidemiology and Biostatistics,

School of Public Health, St Mary's Campus,

Imperial College London, Norfolk Place, London W2 1PG, UK

e-mail: 1.oude-griep@imperial.ac.uk
}

approaches of dietary pattern derivation within a population. Reporting types and absolute intakes of foods and/or food groups may facilitate the identification of a typical dietary pattern that may beneficially influence inflammation.

Keywords Dietary patterns · Cluster analysis . Factor analysis $\cdot$ Reduced rank regression · Diet quality · Inflammation $\cdot$ Atherosclerosis $\cdot$ Epidemiology

\section{Introduction}

Today, the burden of cardiovascular diseases (CVD) has become a worldwide public health problem. For 2010, it has been estimated that CVD was responsible for one in four deaths with a total of 12.9 million deaths globally [1]. Atherosclerosis - one of the most important contributors to the growing burden of CVD - is characterized by the accumulation of lipids and fibrous elements in arteries in which inflammation plays an important role [2]. Inflammatory substances are expressed during the development of atherosclerotic plaques, including the initial disruption of the endothelium by proinflammatory cytokines [3]. C-reactive protein (CRP), an acute-phase reactant, is currently the most validated inflammatory biomarker and is synthesized primarily by hepatocytes in response to cytokines, e.g., interleukin-6 (IL-6), IL-1, and tumour necrosis factor- $\alpha$ $(\mathrm{TNF}-\alpha)[2,3]$. Research interest has therefore expanded toward studying the impact of modifiable factors, such as diet, on inflammatory substances as early markers of atherosclerosis.

Nutrition research was focused traditionally on preventing nutrient deficiencies, but in the 1970s the nutrient-based approach was applied to study the prevention of chronic diseases. With regard to CVD for example, there has been extensive research interest on the beneficial effects of isolated antioxidant vitamins. Prospective cohort studies observed strong inverse associations between intake of antioxidant vitamins and the risk of CVD [4, 5]; however, large 
intervention studies using isolated vitamin supplements could not replicate these positive findings [6, 7]. Nutrients are generally strongly correlated with their dietary sources and the magnitude of the effects of single nutrients on CVD may be too small to detect significant differences beyond confounding and measurement error. Nutrition research has therefore shifted towards studying the synergistic effects of complex whole foods and dietary patterns, acknowledging the reality of the human diet and its usefulness in translating science into dietary recommendations $[8,9]$. The dietary pattern approach provides a comprehensive understanding of studying complex chronic diseases, such as inflammation and endothelial dysfunction, influenced by multiple dietary factors.

Despite the rapid advances in nutritional epidemiology during the last decades, the role of diet in the aetiology of atherosclerosis is still poorly understood. Evidence linking empirically derived dietary patterns and diet quality scores with markers of inflammation and endothelial dysfunction has emerged since 2001 [10••]. The findings of cohort studies published through 2010 on dietary patterns and markers of inflammation and endothelial dysfunction have been summarized recently $[10 \bullet \cdot$. These reviews concluded that previously published cohort studies on dietary patterns and markers of inflammation originated primarily from the United States, were cross-sectional in design, with CRP as the most extensively examined inflammatory marker, and used different approaches to derive dietary patterns [10••]. The different approaches used for defining dietary patterns, however, have influenced the results and interpretation [11•]. This review summarizes the most recently published studies (from January 2011 until January 2013) in nutritional research on associations between empirically derived dietary patterns and diet quality scores with markers of inflammation and endothelial function by considering various approaches of operationalizing dietary patterns.

\section{Differences in Approaches of Dietary Pattern Derivation}

During the past decade, dietary patterns analysis has been applied in many population studies generally based on selfreported food frequency questionnaire data [12]. Exploratory (a posterior or empirical) and hypothesis-oriented (a priori) approaches have been used to derive dietary patterns. Rather than being superior to one or the other, these approaches are designated to answer different study questions [11•, 12-14].

Cluster and factor analyses are two statistical approaches that are mostly used for a posteriori pattern derivation. These approaches encompass important differences; cluster analysis groups individuals based on similarities in their dietary intake, whereas factor analysis identifies highly correlated food or food groups and scores individuals on those factors [11•]. However, a posteriori dietary patterns derived by these two approaches are largely data-driven which result in dietary patterns characteristic only for the population under investigation. Therefore, they may not well explain the variance between dietary patterns and health outcomes and may fail to be good predictors of health [13].

Reduced rank regression (RRR) is a relatively newly applied statistical approach in nutritional epidemiology for dietary pattern derivation [15]. Reduced rank regression identifies foods or food groups in a study population that explain the maximized variation of a priori selected set of responses, e.g., nutrients that have been consistently linked to disease etiology. By integrating a priori knowledge in a posteriori dietary patterns derivation, RRR may serve as a better disease predictor. For all a posteriori derived dietary patterns, however, the lack of generalizability across study populations and health outcomes remains an important weakness.

Score-based dietary patterns are based on a priori knowledge of a set of dietary components $[11 \bullet, 13,14]$. For each individual, a summary index is calculated by crediting higher intake of foods or food-groups considered as healthy, but penalizing overconsumption of those unhealthy [11•]. Various dietary indices have been developed to assess overall diet quality, such as the "Healthy Eating Index," "adherence to the Mediterranean diet score," and "Dietary Guidelines Adherence Index" [11 $, 13,14]$. Hypothesis-oriented scores based on the prevailing evidence of diet-disease associations may fit better for analytic purposes. Selection of dietary components to be included in the index is complicated; results may be explained by an emphasized single component or be attenuated by including irrelevant dietary components. Diet quality indices may differ slightly across countries given differences in Dietary Guidelines, but generally the evidence is consistent regarding the dietary components to encourage or to limit. Diet quality indices allow comparability across cohorts as the scoring is not driven by the population but are limited in capturing their characteristic dietary components.

\section{Methods}

A literature search of the PubMed database of the U.S. National Library of Medicine was conducted to identify relevant studies evaluating associations between empirically derived dietary patterns, diet quality scores, and markers of inflammation. Combinations of the terms "dietary patterns," "food patterns," "factor analysis," "cluster analysis," "principal component analysis," "reduced rank regression," diet quality," "diet scores," "inflammation," "C-reactive protein," and "endothelial function" were used. The search was limited to English-written publications of observational studies published between January 2011 and January 2013. The titles and/or abstracts from the retrieved articles were reviewed to evaluate whether they should be included. Reference lists 
from selected articles also were reviewed for identification of any additional papers not retrieved via the PubMed search. Finally, eight studies published since 2010 were included in this review and are summarized according to the used approach of dietary pattern derivation in Table 1 .

\section{Dietary Patterns and Markers of Inflammation}

\section{Factor Analysis}

Factor analysis or principal component analysis most often were used to derive dietary patterns. Among 9,545 Japanese middle-aged participants of the J-MICC study (2011), participants with higher scores of a healthy dietary pattern had lower CRP concentrations (logarithmically transformed CRP of $0.40 \mathrm{mg} / \mathrm{l}$ in men and $0.29 \mathrm{mg} / \mathrm{l}$ in women) compared with those with lower scores $(0.45 \mathrm{mg} / \mathrm{l}$ in men, $P$ for trend 0.01 ; $0.30 \mathrm{mg} / \mathrm{l}$ in women, $P$ for trend 0.06 in women) [16]. A healthy dietary pattern was characterized by high factor loadings for fruits and vegetables, but no absolute intakes were presented [16]. In 3,978 Chinese men of the SMH Study (2012), participants with a dietary pattern high in fruits, but low in vegetables, had a lower prevalence of elevated CRP, which was defined as $>3 \mathrm{mg} / \mathrm{l}$ (odds ratio (OR) for top vs. lowest quintile: $0.68 ; 95 \%$ confidence interval (CI) 0.46-0.99) [17]. In contrast, a higher prevalence of elevated CRP was found for participants with a high score for an unhealthy dietary pattern high in meat (OR for top vs. lowest quintile: 1.34; $95 \%$ CI 0.91-1.99). A vegetable dietary pattern was not related to the prevalence of CRP [17]. In 981 middle-aged German men of the MONICA/KORA Augsburg surveys (2011), an unhealthy dietary pattern high in meat and beer was strongest correlated to CRP (Spearman correlation coefficient $r=0.24$ ) but less with IL-6 $(r=0.19)$ and IL-18 $(r=0.11)$ [18]. These results have to be interpreted with caution, because correlation coefficients were not adjusted for potential confounders.

In 2,736 relatively young U.S. adults of the CARDIA study (2012), the long-term influences of dietary patterns on $F_{2}$ isoprostanes were studied [19••]. $\mathrm{F}_{2}$-isoprostanes, a measure of oxidative damage, are suggested as predictors of coronary artery calcification and are involved in the early development of coronary artery disease [20]. At baseline, a diet high in fruits (average of 3.0 servings per day) and vegetables diet (average of 1.8 servings of green and 0.6 of yellow vegetables) was inversely associated with average (top vs. lowest quintile $P$ for trend $<0.01$ ) and changes (top vs. lowest quintile $P$ for trend $<0.01)$ in $\mathrm{F}_{2}$-isoprostanes concentrations after more than 15 years of follow-up [19••]. A dietary pattern high in meat (average of 3.0 servings of red meat per day) was positively associated with $\mathrm{F}_{2}$-isoprostanes ( $P$ for trend $<0.0001)$. Dietary patterns derived by factor analysis showed that a diet high in fruits and vegetables was associated inversely with concentrations of CRP and other inflammatory markers. Because average intakes of food groups were only reported in the CARDIA study, it is not possible to compare dietary patterns across studies. It is likely that similarly "labelled" dietary patterns differ largely in their food composition; this limits the comparisons across populations and the potential to define a dietary pattern that may beneficially influence concentrations of inflammatory markers.

\section{Cluster Analysis}

Two cross-sectional studies used cluster analysis to divide participants into groups of comparable dietary patterns. Hlebowicz et al. (2011) derived six dietary patterns characteristic for 4,999 Swedish men and women from the MDC study and found no associations between dietary patterns and CRP [21]. In women, white blood cell (WBC) count was inversely associated with a "fiber-rich bread" dietary pattern (OR for top vs. lowest quartile: 0.5 ; $95 \%$ CI $0.33-0.76$ ) and positively associated with dietary patterns characterized by "milk fat" (OR for top vs. lowest quartile: 1.39; $95 \%$ CI 0.97-1.98) and "sweets and cakes" (OR for top vs. lowest quartile: 1.25; $95 \%$ CI 0.96-1.63). Inverse associations of a "low-fat high-fiber" dietary pattern with lipoprotein-associated phospholipase $\mathrm{A}_{2}$ (Lp-PLA $\mathrm{A}_{2}$ ) mass were found for men (OR for top vs. lowest tertile: $0.62 ; 95 \%$ CI 0.40-0.96) and women (OR for top vs. lowest tertile: 0.69 ; $95 \%$ CI 0.54-0.87). Lp-PLA 2 mass was positively associated with a dietary pattern characterized by "milk fat" in men (OR for top vs. lowest tertile: 1.50; $95 \% \mathrm{CI}$ 1.10-2.05) and by "sweets and cakes" in women (OR for top vs. lowest tertile: 1.29 ; $95 \%$ CI 1.02-1.62) [21]. 1,751 U.S. elderly participants of the Health ABC study (2012) were divided in six dietary patterns with comparable concentration of CRP (range 1.4-1.9 $\mu \mathrm{g} / \mathrm{ml}$ ) and of tumor necrosis factor- $\alpha$ (TNF- $\alpha$; range $2.7-3.2 \mathrm{pg} / \mathrm{ml}$ ) [22]. Participants with a healthy dietary pattern $(n=319)$, however, had significantly lower interleukin-6 (IL-6) concentrations of $1.7 \mathrm{pg} / \mathrm{ml}$ compared with those with dietary patterns characterized by "sweets and desserts" of $1.9 \mathrm{pg} / \mathrm{ml}(n=289 ; P \leq 0.05)$ or by "high-fat dairy" of $1.9 \mathrm{pg} / \mathrm{ml}(n=570 ; P \leq 0.05)$ [22]. Between these two study populations, two dietary patterns appeared similarly named "sweets and cakes/desserts" and "high-fat dairy products/milk fat" clusters; however, absolute intakes and comprising foods or food groups were not presented.

\section{Reduced Rank Regression}

Reduced rank regression was used in two cohort studies to derive dietary patterns. In the aforementioned study among 981 middle-aged men of the MONICA/KORA Augsburg cohort (2011), an RRR-derived western dietary pattern characterized by high intakes of meat and beer and low intakes of vegetables, fruits, wholemeal bread, nuts, and tea was 


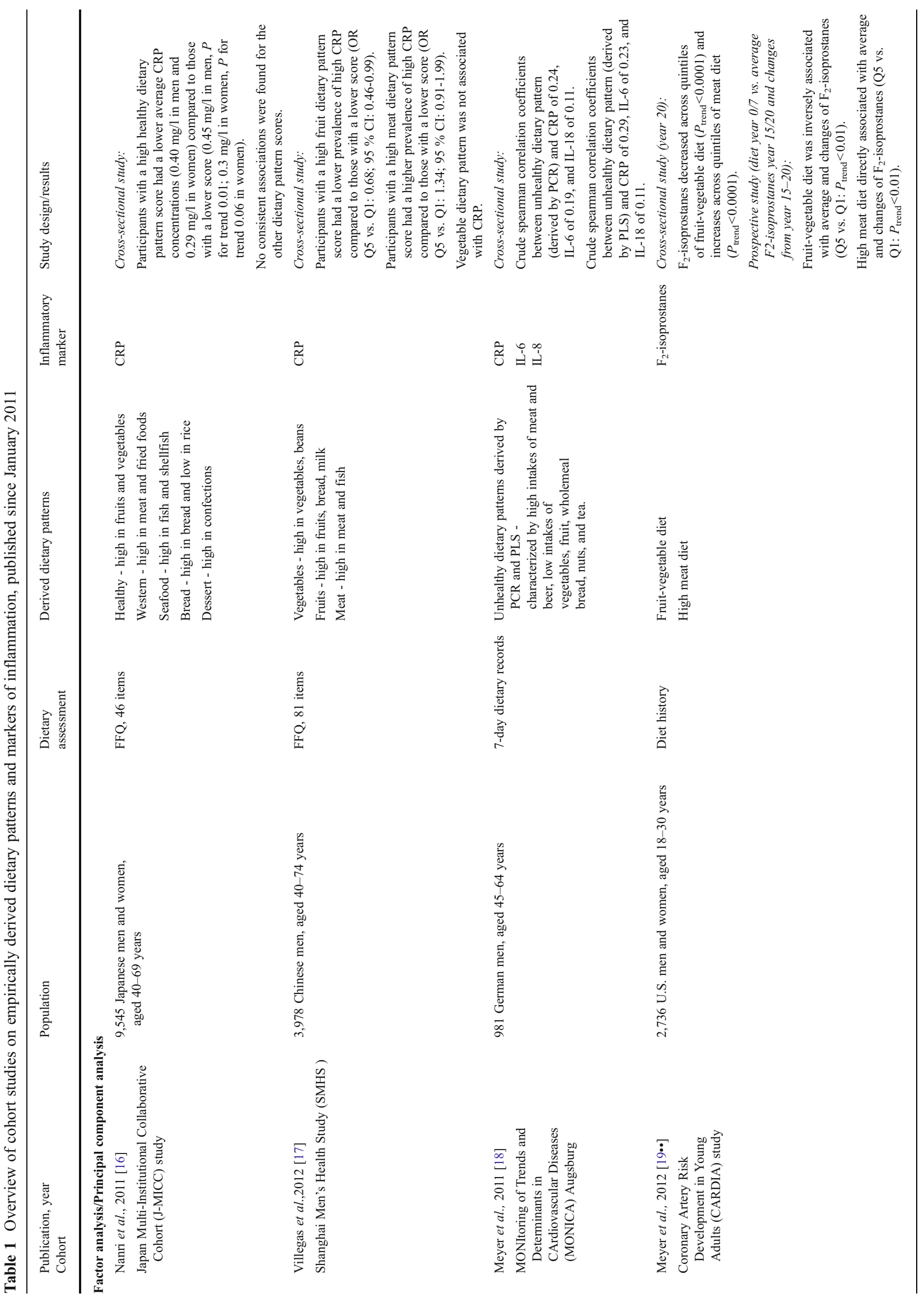




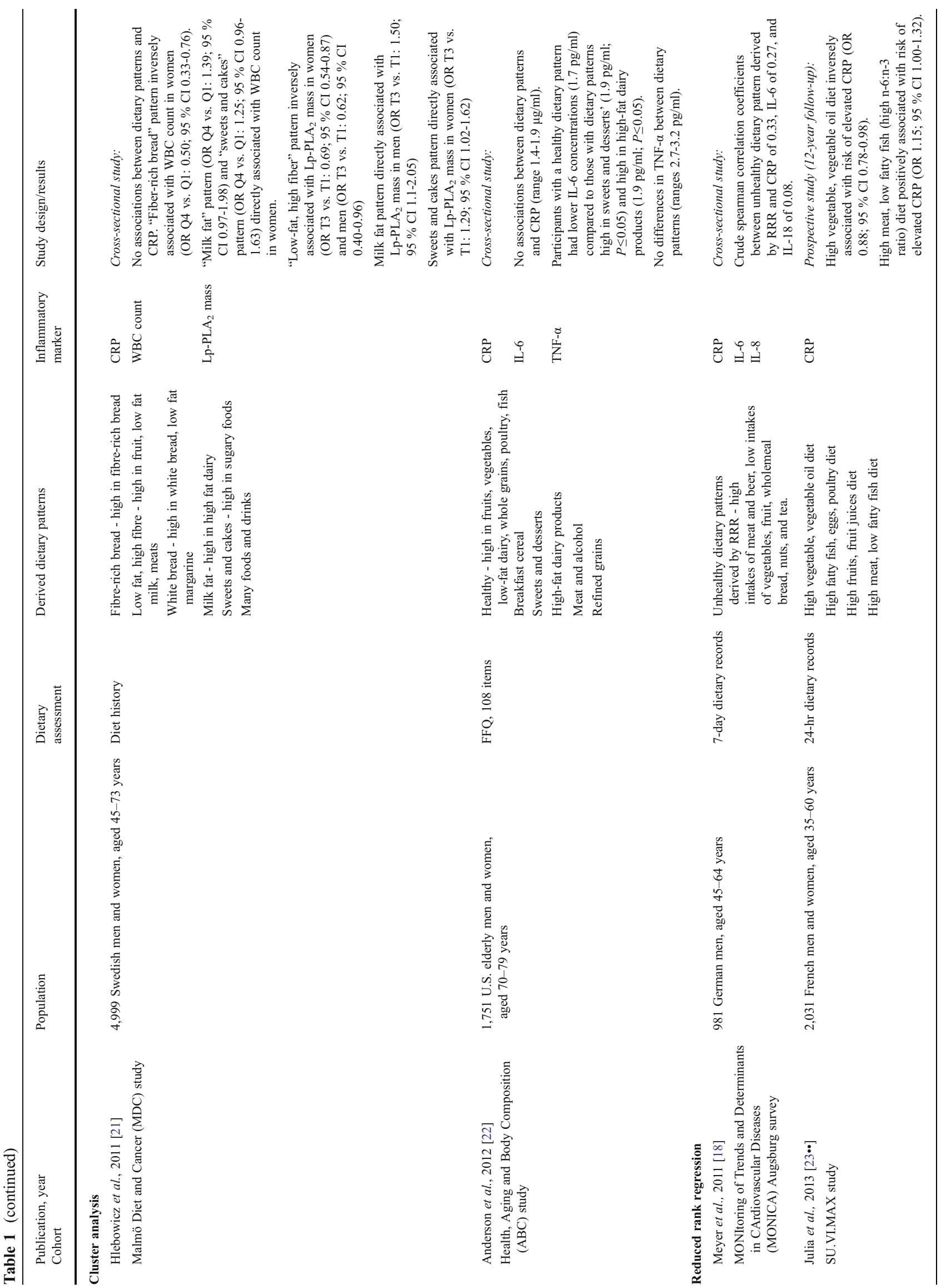




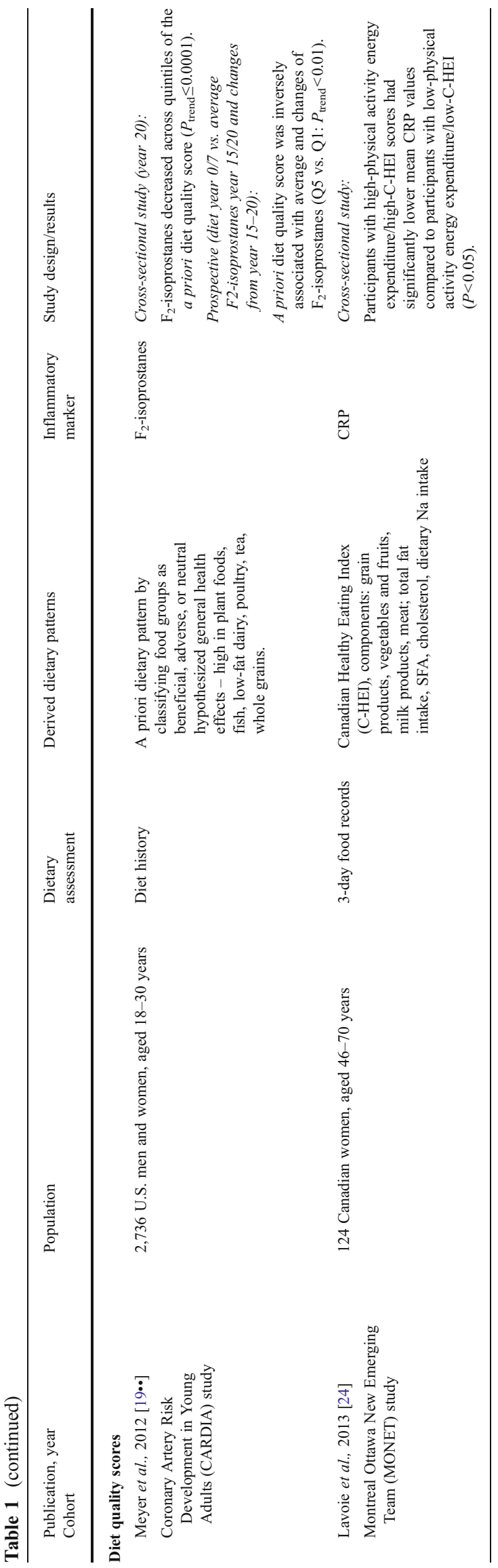

positively correlated with CRP $(r=0.33)$ and IL-6 $(r=0.27)$ but poorly with IL-18 $(r=0.08)$ [18]. In 2,031 middle-aged men and women from the SU.VI.MAX study (2013), the long-term relationship between dietary patterns derived by RRR and CRP has been investigated [23・•]. Nutrients that have been consistently associated with inflammation in previous literature were chosen as responses for RRR; i.e., several poly unsaturated fatty acids and antioxidant micronutrients. A baseline dietary pattern high in vegetables, olive oil, and vegetable oil was associated with a lower risk of elevated CRP of $>3 \mathrm{mg} / \mathrm{l}$ after 12 years of follow-up (OR top vs. lowest tertile 0.69; $95 \%$ CI 0.49-0.95). Contrasting but not significant findings were observed for an unhealthy diet high in processed and organ meat, poultry, eggs, and low in fatty fish (OR top vs. lowest tertile 1.21; $95 \%$ CI 0.87-1.67) [23••]. However, baseline CRP was not measured, limiting the exclusion of participants with elevated CRP at baseline, the adjustment for baseline concentrations, and the assessment of long-term changes in CRP. By including prior knowledge on the link between diet and inflammation, RRR derived dietary patterns may be more useful to detect associations with inflammatory markers.

\section{Diet Quality Scores}

Only two recently published cohort studies were identified using diet quality scores. The Healthy Eating Index adapted for the Canadian population (C-HEI) was used among 124 Canadian middle-aged women of the MONET study (2013) [24]. The C-HEI is created based on consumed servings of foods (e.g., grain products, fruits and vegetables, dairy, and meat), levels of nutrient intake (including total fat, saturated fatty acids, cholesterol, and sodium), and the variety in total food consumption. Participants $(n=33)$ with a high-physical activity energy expenditure level $(>958 \mathrm{kcal} / \mathrm{d})$ and high C-HEI score (>83.3 of 100) had significantly lower CRP concentrations (logarithmically transformed CRP of $0.26 \mathrm{mg} / \mathrm{l})$ than those with a low-physical activity energy expenditure level and low C-HEI $(0.39 \mathrm{mg} / \mathrm{l})$ [24]. The $\mathrm{C}$-HEI, however, includes nutrients in the score system and no distinction is made between whole or refined grain products or (saturated) fat content of dairy and meats. These factors were taken into account in the a priori diet quality score used in the aforementioned CARDIA study (2012) [19••]. This score considered hypothesized general health effects of foods or food groups as well as quintile levels of intake. A healthy dietary pattern with high average daily intakes of fruits (2.5 servings), vegetables ( 1.6 servings of green and 0.5 of yellow vegetables), whole grains (2.2 servings), and seeds and nuts (1.9 servings) was inversely associated cross-sectionally with average (top vs. lowest quintile $P$ for trend $<0.0001$ ) and prospectively with changes (top vs. lowest quintile $P$ for trend $<0.01)$ of $\mathrm{F}_{2}$-isoprostane concentrations [19••]. 


\section{Conclusions}

Overall, the results of recently published cohort studies support findings of previous cross-sectional studies suggesting that consuming a healthy type of diet characteristically abundant in fruit and vegetables is associated with lower concentrations of CRP and other inflammatory markers. In contrast, unfavorable associations were found between eating a western unhealthy type of diet (e.g., high in meat) and inflammatory markers. These findings were observed in studies that derived dietary patterns by means of factor analysis, RRR, and a priori definitions, but not in those using cluster analyses. Due to the use of different statistical procedures, the defined dietary patterns appear similar but may comprise different accompanying foods or individuals characteristic for the population under investigation. Comparisons of absolute intakes of foods or food groups between studies are hindered since only one study reported absolute intakes [19••], other studies reported dietary pattern composition as energy percentages of highly contributing food sources [21] or nutrients [22], factor loadings [16-18, 23••], or scores for individual dietary components [24].

This also limits judgement of whether a healthy dietary pattern may have clinically relevant effects on inflammatory markers. Future studies therefore need to describe clearly derived dietary patterns by presenting absolute intakes of foods and food groups to enable comparisons between studies and to characterise a typical dietary pattern that may beneficially influence inflammation.

Cluster and factor analyses are useful approaches for exploring characteristic dietary patterns of a specific population and hypothesis generation in relation to health outcomes; however, such dietary patterns are only crude indicators of a (un)healthy dietary pattern. Because scientific knowledge is integrated in RRR and a priori scores to derive dietary patterns studies, it is not surprising that these approaches found stronger associations with inflammatory markers. Given the synergistic effects of complex whole foods and the call for food-based dietary guidelines [25], it is important to operationalize dietary patterns based on foods instead of nutrients. Such an approach has been applied in the CARDIA study in which an a priori dietary patterns was defined by rating foods or food groups as beneficial, adverse, or neutral according to hypothesized health effects $[19 \bullet \bullet]$. In contrast to other diet quality scores, this approach also takes into account the quantity of food consumption. As shown by two cohort studies $[18,19 \bullet \cdot]$, it is worth comparing dietary patterns derived by various approaches to understand their different characteristics and their results related to inflammatory markers.

Two recently published prospective cohort studies on dietary patterns and inflammation added important knowledge to this research area confirming the beneficial cross-sectional associations found between a healthy type of diet and inflammatory markers $[19 \bullet \bullet, 23 \bullet \bullet]$. Such studies are important from an aetiological point of view because pathophysiological processes underlying the inflammation and the development of atherosclerosis are in constant continuation during the life-course. Little evidence is available for the impact of long-term adherence to a healthy dietary pattern using repeated measurements and changes in inflammation and atherosclerosis throughout the life span. To the best of our knowledge, only one relatively long-term, randomized, controlled trial of 2 years has been published, which found that patients with the metabolic syndrome adherent to a Mediterranean-style of diet had a significant reduction of CRP compared with the control group consuming a prudent diet [26••]. With regards to atherosclerosis and life-course analysis, a recently published prospective cohort study following 373 healthy participants since age 13 years for an of average 24 years suggested a favorable association between adherence to a Mediterranean diet throughout adolescence and early adulthood and stiffer carotid arteries in adulthood $[27 \bullet \bullet]$. The research question of whether consuming a healthy dietary pattern from younger age delays atherogenesis and lowers the number of CVD cases at later life is a challenge for future nutritional epidemiology research.

Acknowledgments The authors thank Professor P. Greenland for helpful advice in reading the manuscript. L.M. Oude Griep and Q. Chan are supported by grants R01 HL50490 and R01 HL084228 from the National Heart, Lung, and Blood Institute, National Institutes of Health (Bethesda, MD).

Conflict of Interest Linda M. Oude Griep declares she has no conflict of interest.

Huifen Wang declares she has no conflict of interest.

Queenie Chan declares she has no conflict of interest.

\section{References}

Papers of particular interest, published recently, have been highlighted as

- Of importance

-• Of major importance

1. Lozano R, Naghavi M, Foreman K, et al. Global and regional mortality from 235 causes of death for 20 age groups in 1990 and 2010: a systematic analysis for the Global Burden of Disease Study 2010. Lancet. 2012;380:2095-128.

2. Ross R. Atherosclerosis-an inflammatory disease. N Engl J Med. 1999;340:115-26.

3. Libby P. Inflammation in atherosclerosis. Nature. 2002;420:868-74.

4. Knekt P, Ritz J, Pereira MA, et al. Antioxidant vitamins and coronary heart disease risk: a pooled analysis of 9 cohorts. Am J Clin Nutr. 2004;80:1508-20.

5. Ye Z, Song H. Antioxidant vitamins intake and the risk of coronary heart disease: meta-analysis of cohort studies. Eur J Cardiovasc Prev Rehabil. 2008;15:26-34. 
6. Sesso HD, Buring JE, Christen WG, et al. Vitamins $\mathrm{E}$ and $\mathrm{C}$ in the prevention of cardiovascular disease in men: the Physicians' Health Study II randomized controlled trial. JAMA. 2008;300:2123-33.

7. Vivekananthan DP, Penn MS, Sapp SK, et al. Use of antioxidant vitamins for the prevention of cardiovascular disease: metaanalysis of randomised trials. Lancet. 2003;361:2017-23.

8. Stanner SA, Hughes J, Kelly CN, Buttriss J. A review of the epidemiological evidence for the 'antioxidant hypothesis'. Public Health Nutr. 2004;7:407-22.

9. Jacobs DR, Tapsell LC. Food synergy: the key to a healthy diet. Proc Nutr Soc. 2013:1-7.

10. • Ahluwalia A, Andreeva V, Kesse-Guyot E, Hercberg S. Dietary patterns, inflammation and the metabolic syndrome. Diab Metab. 2012, In press. This review summarizes findings of cohort studies on dietary patterns and markers of inflammation and endothelial dysfunction until around 2011.

11. - Reedy J, Wirfalt E, Flood A, et al. Comparing 3 dietary pattern methods-cluster analysis, factor analysis, and index analysis-with colorectal cancer risk: the NIH-AARP diet and health study. Am J Epidemiol. 2010;171:479-87. This cohort study on dietary patterns and colorectal cancer illustrates that findings can vary depending on method of dietary pattern derivation by comparing cluster, factor, and index analysis. The differences in methods and interpretation are explained clearly.

12. Newby PK, Tucker KL. Empirically derived eating patterns using factor or cluster analysis: a review. Nutr Rev. 2004;62:177-203.

13. Moeller SM, Reedy J, Millen AE, et al. Dietary patterns: challenges and opportunities in dietary patterns research an experimental biology workshop, April 1, 2006. J Am Diet Assoc. 2007;107:1233-9.

14. Wirt A, Collins CE. Diet quality - what is it and does it matter? Public Health Nutr. 2009;12:2473-92.

15. Hoffmann K, Schulze MB, Schienkiewitz A, et al. Application of a new statistical method to derive dietary patterns in nutritional epidemiology. Am J Epidemiol. 2004;159:935-44.

16. Nanri H, Nakamura K, Hara M, et al. Association between dietary pattern and serum C-reactive protein in Japanese men and women. J Epidemiol. 2011;21:122-31.

17. Villegas R, Xiang YB, Cai H, et al. Lifestyle determinants of Creactive protein in middle-aged, urban Chinese men. Nutr Metab Cardiovasc Dis. 2012;22:223-30.

18. Meyer J, Doring A, Herder C, et al. Dietary patterns, subclinical inflammation, incident coronary heart disease and mortality in middle-aged men from the MONICA/KORA Augsburg cohort study. Eur J Clin Nutr. 2011;65:800-7.

19. •- Meyer KA, Sijtsma FP, Nettleton JA, et al. Dietary patterns are associated with plasma $\mathrm{F}_{2}$-isoprostanes in an observational cohort study of adults. Free Rad Biol Med. 2012: in press. In this prospective cohort study on dietary patterns and $F_{2}$-isoprostanes used both a posteriori and a priori dietary pattern derivation methods. The a priori dietary pattern took into account previous evidence on health effects and quantity of food intake.

20. Gross M, Steffes M, Jacobs Jr DR, et al. Plasma F2-isoprostanes and coronary artery calcification: the CARDIA Study. Clin Chem. 2005;51:125-31.

21. Hlebowicz J, Persson M, Gullberg B, et al. Food patterns, inflammation markers and incidence of cardiovascular disease: the Malmo diet and cancer study. J Int Med. 2011;270:365-76.

22. Anderson AL, Harris TB, Tylavsky FA, et al. Dietary patterns, insulin sensitivity and inflammation in older adults. Eur J Clin Nutr. 2012;66:18-24.

23. • Julia C, Meunier N, Touvier M, et al. Dietary patterns and risk of elevated C-reactive protein concentrations 12 years later. Br J Nutr. 2013:1-8. This is the first prospective cohort study among 2,031 French middle-aged participants on dietary patterns derived by reduced rank regression and $C$-reactive protein. After 12 years of follow-up, a healthy high vegetable diet was inversely associated with the risk of elevated C-reactive protein; an unhealthy, high meat dietary pattern was positively associated with risk of elevated CRP.

24. Lavoie ME, Faraj M, Strychar I, et al. Synergistic associations of physical activity and diet quality on cardiometabolic risk factors in overweight and obese postmenopausal women. Br J Nutr. 2013;109:605-14.

25. Mozaffarian D, Ludwig DS. Dietary guidelines in the 21 st century-a time for food. JAMA. 2010;304:681-2.

26. - Esposito K, Marfella R, Ciotola M, et al. Effect of a mediterranean-style diet on endothelial dysfunction and markers of vascular inflammation in the metabolic syndrome: a randomized trial. JAMA. 2004;292:1440-6. In this randomized controlled trial among 180 patients with the metabolic syndrome, patients' adherent to a Mediterranean-style of diet for 2 years had a significant reduction of CRP compared to the control group consuming a prudent diet.

27. ••van de Laar RJ, Stehouwer CD, van Bussel BC, et al. Adherence to a Mediterranean dietary pattern in early life is associated with lower arterial stiffness in adulthood: the Amsterdam Growth and Health Longitudinal Study. J Int Med. 2013;273:79-93. This prospective cohort study followed 373 healthy participants since age 13 for on average 24 years and observed a favourable association between adherence to a Mediterranean diet throughout adolescence and early adulthood and stiffer carotid arteries in adulthood. 